Voix et Images

volxetimages

\title{
Quand Refus global devient « Refus global »
}

Les conditions de la réception à la fin des années 1940

When Refus Global Became 'Refus Global'

Conditions of Reception in the Late 1940s

Cuando Refus global se transforma en 'Refus global'

Las condiciones de su recibimiento a finales de los años 1940

\section{Sophie Dubois}

Volume 41, numéro 2 (122), hiver 2016

La révolution littéraire des années 1940 au Québec

URI : https://id.erudit.org/iderudit/1036939ar

DOI : https://doi.org/10.7202/1036939ar

Aller au sommaire du numéro

\section{Éditeur(s)}

Université du Québec à Montréal

\section{ISSN}

0318-9201 (imprimé)

1705-933X (numérique)

Découvrir la revue

\section{Citer cet article}

Dubois, S. (2016). Quand Refus global devient « Refus global " : les conditions de la réception à la fin des années 1940. Voix et Images, 41(2), 95-105.

https://doi.org/10.7202/1036939ar

\section{Résumé de l'article}

Souvent retenu comme l'amorce de la modernité culturelle québécoise, le manifeste automatiste Refus global est examiné dans cet article du point de vue de sa première réception (1948-1949). Sont interrogés les critères sociohistoriques et esthétiques privilégiés à la fin des années 1940 ayant permis à l'oeuvre d'acquérir le statut de texte fondateur grâce à un resserrement du discours critique autour du texte éponyme, au détriment des autres composantes du recueil original. Une étude de la centaine d'articles formant la première réception révèle que cinq facteurs principaux jouent un rôle dans la lecture faite de l'oeuvre et dans le statut qui lui est octroyé : la matérialité du recueil original, l'état des champs artistiques, les critères de légitimité établis par la critique, la réaction étatique et l'attitude des auteurs envers la critique. Chacun de ces facteurs est analysé et historicisé par l'auteure, qui montre comment, à l'époque, une oeuvre recueillistique et pluridisciplinaire comme Refus global bouleverse l'horizon d'attente, entre autres en allant à contresens de la logique moderniste, malgré ce que la critique en a retenu. L'étude de la première réception permet ainsi à la fois d'expliquer la réduction du discours critique ayant conduit à la consécration du texte éponyme " Refus global » et de mettre en évidence d'autres lectures, faites avant la constitution du discours dominant ou en parallèle avec celui-ci, susceptibles de réévaluer le statut aujourd'hui attribué à l'oeuvre. 
SOPHIE DUBOIS

Université de la Sarre (Allemagne)

Refus global n'est pas «Refus global». C'est-à-dire que, malgré ce qu'en a retenu la critique, le recueil collectif et pluridisciplinaire paru en 1948 ne se réduit pas à son texte éponyme. À sa parution, Refus global est en effet composé de neuf textes de Paul-Émile Borduas, de Claude Gauvreau, de Françoise Sullivan, de Bruno Cormier et de Fernand Leduc, accompagnés de photographies prises par Maurice Perron et d'une couverture réalisée par Jean-Paul Riopelle. À l'intérieur de cet ensemble, «Refus global» occupe certes une position prédominante: signé collectivement par les membres du groupe, il est à l'origine de la publication'², qu'il ouvre et à laquelle il donne son titre. Aussi s'impose-t-il d'emblée comme le texte phare du recueil. Or cette prédominance justifie-t-elle, à elle seule, le statut de "mythe originaire» de la modernité québécoise ${ }^{3}$ aujourd'hui conféré au texte éponyme et constitue-t-elle, par le fait même, la seule raison expliquant l'effacement presque complet des autres composantes du recueil dans l'histoire culturelle québécoise?

S'interroger sur les facteurs de la réception critique qui, dès la parution de Refus global, ont conduit, d'une part, à l'effacement du recueil et, d'autre part, à la consécration du texte éponyme permet de prendre le pouls du champ culturel de l'époque. Dans cette optique, cinq obstacles à la réception du recueil Refus global ont été retenus, lesquels révèlent les critères et les tensions qui constituaient la dynamique du champ entre 1948 et 1950.

1 Cet article est issu de mes recherches doctorales, lesquelles ont reçu l'appui financier du Conseil de recherches en sciences humaines du Canada (CRSH). Il résume la deuxième partie de ma thèse; j'y renvoie le lecteur pour plus de détails: Sophie Dubois, Quand Refus global devient «Refus global». L'histoire d'une réception partielle, thèse de doctorat, Montréal, Université de Montréal, 2014, 658 f.

2 La décision de joindre d'autres productions au texte collectif est en effet postérieure à l'écriture de «Refus global». Selon Gauvreau, ce serait Borduas qui aurait cru «bon d'étoffer l'acte collectif». Voir Claude Gauvreau, "L'épopée automatiste vue par un cyclope», La Barre du jour, ns 17-20, janvier-août 1969, p. 71.

3 Voir Yvan Lamonde et Esther Trépanier (dir.), L'avènement de la modernité culturelle au Québec, Québec, Institut québécois de recherche sur la culture, 1986, p. 11. Les textes réunis dans cet ouvrage visent notamment à nuancer ce statut octroyé à «Refus global». 


\section{LA MATÉRIALité : ENTRAVE À LA LECTURE}

Tiré à quatre cents exemplaires, le recueil Refus global se présente à ses premiers lecteurs sous une forme artisanale: il est composé d'un ensemble de textes tapuscrits contenus dans des cahiers séparés, puis rassemblés dans une couverture de carton sans reliure. Outre la jaquette qui les regroupe, seul le paratexte (tables des matières et des illustrations, d'ailleurs incomplètes) offre, sur le plan matériel, une forme d'unité à cet ensemble. Ainsi, les commentateurs se heurtent à une première difficulté: celle de la définition de l'objet qu'ils ont entre les mains. Plusieurs des critiques consacrent alors une partie - voire la totalité - de leur article à décrire et à commenter la matérialité du recueil ${ }^{4}$. Certains hésitent d'ailleurs à qualifier Refus global de «livre»: André Lecompte du Petit Journal désigne l'œuvre des automatistes comme "leur livre ou leur j'sais pas quoi ${ }^{5}$ ", alors que Roger Duhamel de MontréalMatin s'interroge: "Un livre? Plus exactement un certain nombre de feuilles miméographiées ${ }^{6} . »$

L'hétérogénéité des composantes et l'aspect artisanal du recueil rendent en effet difficile la saisie de l'œuvre dans son ensemble en fonction d'une lecture unifiée, d'autant que, comme le souligne Patrick Imbert, "peu de textes "contestataires" ont été publiés sous forme de livres ${ }^{7} »$ au Québec avant Refus global. Les catégories du manifeste artistique ${ }^{8}$ ou du livre d'artiste ${ }^{9}$, par exemple, sont inexistantes et ne peuvent fournir un cadre d'expériences préalables à même de faciliter la prise en charge de Refus global par la critique. La matérialité de l'œuvre - premier contact du lecteur avec celle-ci - a donc ici un impact considérable sur sa réception. Les critiques, confrontés à cette esthétique livresque inhabituelle, s'attardent dès lors davantage au contenant qu'au contenu, en soulignant notamment l'inadéquation du support au message: "La souplesse d'un livre ou d'une forte brochure eût mieux convenu à ce message. Tel qu'il est présenté, il nous paraît éphémère et il accroît les

4 «It has been issued in the form of a small portfolio, containing a number of mimeographed articles on painting, drama, poetry and the dance, also a few illustrations printed on coated paper», écrit par exemple Donald W. Buchanan («New Books on the Art. Refus global», Canadian Art, Christmas 1948, p. 86). («Il a été publié sous la forme d'un petit portfolio contenant un certain nombre d'articles miméographiés sur la peinture, le théâtre, la poésie et la danse, ainsi que quelques illustrations imprimées sur du papier couché.» Je traduis.)

5 André Lecompte, «L'œil en coulisse», Le Petit Journal, 29 août 1948, p. 51.

6 Roger Duhamel, «Notes de lecture. "Refus global”", Montréal-Matin, 14 septembre 1948, p. 4.

7 Patrick Imbert, «Réception des surréalistes québécois», Henri Béhar (dir.), Le livre surréaliste, Lausanne, L'Âge d'Homme, coll. «Mélusine» des Cahiers du Centre de recherches sur le surréalisme, 1981, p. 57.

8 Certes, était paru quelques mois auparavant le manifeste Prisme d'yeux du groupe d'Alfred Pellan, mais sur le plan de la matérialité, celui-ci ne correspond en rien à l'aspect recueillistique et hétérogène de Refus global: il s'agit d'un simple feuillet de circonstance distribué lors d'une soirée d'exposition. Quant aux manifestes surréalistes, André-G. Bourassa soutient qu'ils «étaient introuvables à Montréal » à l'époque, ce que le peu de références qui y sont faites dans la réception du recueil automatiste tend à confirmer. Voir André-G. Bourassa, Surréalisme et littérature québécoise, Montréal, L'Étincelle, 1977, p. 62.

9 L'idée selon laquelle Refus global formerait un «livre d'artiste avant la lettre» est récente; je l'ai notamment défendue dans un article qui comprend certaines réflexions contenues dans cette section: Sophie Dubois, «Refus global ou comment faire les frais d'une présentation», Stéphanie Bernier, Sophie Drouin et Josée Vincent (dir.), Le livre comme art. Matérialité et sens, Québec, Nota bene, coll. «Sciences humaines/littérature», 2013, p. 83-98. 
préventions de ceux qui soupçonnent déjà une plaisanterie ${ }^{10}$.» La lecture du recueil est donc d'abord empêchée par sa propre matérialité, qui interfère avec le contenu et qui, de surcroît, tend à discréditer l'œuvre qualifiée ailleurs de bricolage d'enfant d'école («almost schoolboy's stuff ${ }^{11} »$ ).

Privé d'un horizon d'attente favorable à sa réception, c'est-à-dire en l'absence d'œuvres antérieures susceptibles de préparer la critique à la matérialité artisanale du recueil automatiste, celui-ci bouleverse les schèmes interprétatifs des lecteurs en remettant en question l'idée qu'ils se font généralement du livre comme forme cohérente, linéaire et unifiée. Il n'est alors pas étonnant que la description de l'objet occupe une place non négligeable au début de la réception jusqu'à restreindre le propos à une évaluation, souvent négative, du support. En outre, la matérialité de l'œuvre, composée de cahiers reliés et paginés séparément, légitime une lecture individualisée des composantes, lesquelles peuvent être détachées du recueil, comme ce sera le cas du texte éponyme de Borduas retenu dans l'histoire au détriment de «l'acte collectif» que devait être Refus global.

\section{LA PLURIDISCIPLINARITÉ}

Si la matérialité du recueil entrave sa saisie comme objet cohérent, sa pluridisciplinarité se révèle également problématique dans un contexte où le processus d'autonomisation des disciplines formant le champ culturel n'est pas encore complété.

D'une part, la frontière toujours poreuse entre les divers arts dans le champ culturel rend possible la réception d'une œuvre faisant collaborer des plasticiens, un poète, une chorégraphe, un psychanalyste et un photographe. Le recueil bénéficie en effet de lecteurs critiques qui, grâce à une formation classique et à des intérêts relevant à la fois de la littérature, des arts visuels et de la philosophie, sont aptes à en saisir les diverses composantes et à en apprécier la nature pluridisciplinaire. La polyvalence de ces critiques leur permet de discuter tant les idées que la forme de l'œuvre et d'évaluer en parallèle diverses productions qu'elle contient. Aussi sont-ils à même de traiter à la fois de $l^{\prime}$ « automatisme en peinture et au thêâtre ${ }^{12}$ ", tout en souhaitant " voir le groupe automatiste tendre ses filets du côté des jeunes musiciens ${ }^{13}$ » ou encore peuvent-ils juger que Borduas a un «indiscutable talent de peintre [mais qu']il est moins heureux dans le pamphlet $^{14}{ }^{»}$ ou que les «jeunes [...] valent mieux quand ils se confinent à la peinture ${ }^{15} »$.

Les quelques articles abordant de la sorte la pluridisciplinarité du recueil forment cependant la part congrue de la réception puisque, d'autre part, la tendance

10 Lafcadio, «L'underground de l'esthétique. "Refus global". Manifeste de l'automatisme surrationnel par Paul-Émile Borduas et compagnie», Le Canada, 23 août 1948, p. 4.

11 Guy Jasmin, «Quebec Artist Causes Furor. Fired from Provincial Government School Because of Outspoken Comments», The Windsor Daily Star, 26 novembre 1948, [page éditoriale].

12 [Pierre Saint-Germain], «Écartèlement et jus de tomates. Nos automatistes annoncent la décadence chrétienne et prophétisent l'avènement de l'instinct», Le Petit Journal, 15 août 1948, p. 28.

13 Charles Doyon, «"Refus global”», Le Clairon [Saint-Hyacinthe], 27 août 1948, p. 5.

14 Roger Duhamel, «Notes de lecture. "Refus global"», p. 4.

15 Lafcadio, «L'underground de l'esthétique», p. 4. 
à l'autonomisation et à la singularisation des disciplines complexifie - voire empêche - la saisie de l'œuvre dans son ensemble. En témoigne l'embarras de Rolland Boulanger, critique en arts visuels au Montréal-Matin, devant la tâche de décrire les composantes non artistiques de Refus global: "[Gauvreau] contribue à l'ouvrage par un ensemble de trois... "pièces de théâtre" surréalistes, plutôt courtes, dont je me dispense a priori de parler, laissant aux chroniqueurs autorisés dans la matière d'y appuyer ailleurs. De même l'étude sur la danse de Françoise Sullivan ${ }^{16}$.» L'idée selon laquelle il faut posséder une compétence disciplinaire spécifique - être autorisé dans la matière - pour émettre un jugement sur une œuvre relève de cette nouvelle conscience des critiques spécialisés qui se développe dans les années 1940 alors que, comme le mentionne Michel Biron, « [l]a formation classique ne suffit plus: le savoir se mesure encore à une certaine connaissance générale, mais [...] il faut une connaissance spécialisée pour acquérir une légitimité institutionnelle ${ }^{17}$ ». Dans cette perspective, Refus global se voit réduit, le plus souvent, à son appartenance aux arts visuels. Désigné alors comme le «manifeste des peintres automatistes» ou "d'un groupe de peintres», le recueil perd sa dimension pluridisciplinaire au profit d'une prise en charge par un discours critique spécialisé et d'une inscription dans une histoire disciplinaire spécifique - conditions essentielles pour éviter de sombrer dans l'oubli dans un contexte où les disciplines prennent conscience d'elles-mêmes et développent leur propre expertise et leur propre métadiscours.

\section{RIDICULISATION ET MINORATION EN REACTION À L'ÉCART ESTHÉTIQUE}

À l'inverse du texte de Borduas, qui est lu en fonction du message qu'il contient, sans réelle préoccupation pour sa forme littéraire, les autres composantes trouvent difficilement des commentateurs en raison du trop grand écart esthétique ${ }^{18}$ qu'elles présentent avec les attentes des lecteurs de l'époque. Comme en témoigne l'hésitation de Rolland Boulanger citée plus haut, les critiques éprouvent le plus souvent de la perplexité et un malaise devant cette œuvre qui bouleverse radicalement leurs attentes et leurs schèmes interprétatifs. Afin de pallier ce sentiment d'impuissance, ils usent de divers moyens les exemptant de toute forme approfondie d'herméneutique : simples descriptions objectives de l'œuvre, suspension du jugement ${ }^{19}$, recours à la citation massive ou, plus simplement, entreprise de ridiculisation et de minoration.

16 Rolland Boulanger, «Dynamitage automatiste à la Librairie Tranquille», Montréal-Matin, 9 août 1948, p. 5.

17 Michel Biron, L'absence du maître. Saint-Denys Garneau, Ferron, Ducharme, Montréal, Presses de l'Université de Montréal, coll. «Socius», 2000, p. 111.

18 Voir Hans Robert Jauss, Pour une esthétique de la réception, Paris, Gallimard, coll. «Tel», 1978, p. 59.

19 Gérard Pelletier avoue d'entrée de jeu dans son article: «Si nous avons tardé [à commenter l'œuvre], c'est que le document nous laisse un peu perplexe» et il poursuit ainsi à propos du travail des «jeunes» automatistes: «[a]ussi longtemps qu'on ignore où cette recherche conduit et qu'on peut lui supposer une constance honnête, on est tenté de réserver l'avenir, d'attendre les développements». («Deux âges, deux manières», Le Devoir, 25 septembre 1948, p. 8.) La "suspension prudente du jugement, signale Nathalie Heinich, est une première façon de réagir, pour qui hésite encore entre disqualification d'une singularité aberrante et adhésion à une singularité innovante» (Le triple jeu de l'art contemporain. Sociologie des arts 
Déjà, on l'a vu, l'aspect artisanal du recueil Refus global est perçu par la critique comme une "plaisanterie", comme la preuve d'un manque de sérieux de la part des automatistes. Les idées et la forme qu'il adopte font également l'objet de diverses mises en scène humoristiques, d'anecdotes loufoques, de pastiches ${ }^{20}$ ou de caricatures qui ont pour but - et bien souvent pour effet - de discréditer le recueil et l'automatisme en général au nom d'un «art authentique».

Les critiques, observant l'œuvre à partir de leur «monde rationnel et non initié $^{21}$ ", ne peuvent que constater la distance qui les sépare de l'automatisme, lequel semble appartenir à un autre "monde», irrationnel et fermé, inaccessible, soutiennent-ils, «à qui use normalement de ses facultés ${ }^{22}$ ». Dans le cas de Refus global, l'écart ressenti dépend notamment du niveau d'adhésion des critiques à l'art moderne et aux nouveaux critères de légitimation qu'il implique. À cet égard, un article du Petit Journal s'avère révélateur d'une certaine attitude ambiguë et velléitaire de la part des critiques qui, s'ils connaissent ces nouveaux critères, refusent de reconnaître l'usage qu'en font les automatistes:

Nos automatistes ne sont d'ailleurs pas sûrs d'avoir atteint le nec plus ultra, puisqu'ils viennent d'apprendre qu'un Roumain du nom de Trost a publié récemment un manifeste du «surautomatisme». On ne peut en tout cas pas leur nier un beau désintéressement. Ils ne croient pas au commercialisme et aux honneurs [...] et ils qualifient de poires ceux qui achètent leurs tableaux ${ }^{23}$.

Si le commentateur semble concéder aux automatistes le désintéressement exigé en matière d'art moderne, le ton avec lequel il le fait suggère que, pour lui, ce critère ne fonde pas la légitimité de l'art: au contraire, il rend ridicules les auteurs qui méprisent leurs acheteurs et qui se privent ainsi du fruit de leur travail. Par ailleurs, la triple négative («ne pas»; «nier» et le préfixe «dés-») rend la formulation confuse et semble insinuer, par un effet ironique accentué par l'adjectif «beau», que les automatistes ne seraient peut-être pas si désintéressés... De même, le critère d'originalité, convoqué ici pour discréditer l'automatisme, n'est pas non plus jugé pertinent: l'auteur souligne plutôt l'absurdité de la logique de rupture incessante et de surenchère novatrice exigées par l'art moderne. C'est dans cette même optique qu'un critique du Canada dénonce ces "soi-disant novateurs» et leurs «extravagances modernes ${ }^{24} »$ qui feraient reposer les œuvres sur des artifices conceptuels ou

plastiques, Paris, Éditions de Minuit, coll. «Paradoxe», 1998, p. 188). Or cette suspension du jugement, si elle est "prudente», peut également s'avérer dangereuse car, si le jugement demeure ainsi indéfiniment suspendu, il conduit inévitablement à l'oubli de l'œuvre.

20 Sur les pastiches de Claude Gauvreau par Odette Oligny, voir Sophie Dubois, "Charge et contre-charge. Claude Gauvreau, pastiché et pasticheur», Voix et Images, vol. XXXIX, nº 3, printemps-été 2014, p. 97-109.

21 «Écartèlement et jus de tomates», p. 28.

22 Rolland Boulanger, «Dynamitage automatiste à la Librairie Tranquille», p. 5. Notons au passage que, comme le souligne Nathalie Heinich, l'imputation de folie «revient de façon récurrente pour "inauthentifier" l'art moderne» (Le triple jeu de l'art contemporain, p. 34).

23 «Écartèlement et jus de tomates», p. 28. Sur le «surautomatisme», voir Gherasim Luca et Dolfi Trost, Dialectique de la dialectique, Bucarest, Éditions surréalistes, 1945, 30 p.

24 Lafcadio, «L'underground de l'esthétique», p. 4. 
que Robert LaPalme, dans une caricature intitulée «Ces temps modernes ${ }^{25}$ ", rattache le caractère fluctuant de l'art et des mouvements artistiques à celui des échanges sportifs en présentant côte à côte une nouvelle radiophonique et un article journalistique annonçant respectivement que «Les automatistes cèdent Gauvreau aux Prisme $d^{\prime}$ yeux pour \$3,25\$" et que "le Chicago échange deux joueurs avec le Boston ${ }^{26}$ ". À travers la ridiculisation de Refus global, c'est la logique même de l'art moderne - notamment le caractère éphémère de la nouveauté qui y prévaut - qui est mise en doute par une partie de la critique. Celle-ci, refusant de reconnaître la légitimité de ces nouveaux critères, ne peut concevoir l'art automatiste comme recevable; c'est la nature même d'œuvre d'art «authentique» qui est alors refusée à Refus global et à l'automatisme en général. Dans un contexte d'art moderne où « [h]umilité, désintéressement, originalité, intériorité, inspiration, sincérité, sérieux, rationalité [...] sont les principales valeurs attestant l'authenticité d'un artiste ${ }^{27}$ ", l'automatisme peine à convaincre la critique de son sérieux et de son honnêteté. Or, comme le signale Heinich, ces deux critères sont primordiaux dans un milieu où l'art moderne ne peut compter sur de véritables «spécialistes»:

Si l'originalité et l'inspiration sont davantage privilégiées à l'intérieur du monde de l'art, [...] sincérité et raison [...] sont souvent invoqué[e]s par les profanes pour disqualifier des propositions auxquelles ils n'accordent même pas le sérieux qui permettrait de s'interroger sur leur originalité ou leur inspiration ${ }^{28}$.

Cette entreprise $d^{\prime}$ «inauthentification», qui passe notamment par la moquerie et par la minoration, touche principalement les composantes marginales de Refus global et, au premier chef, les textes de Claude Gauvreau, dont on dit qu'il a «beaucoup d'avenir dans la loufoquerie 29 ». Or, comme le mentionnent Chaïm Perelman et Lucie Olbrechts-Tyteca, la ridiculisation est «une façon de condamner une conduite excentrique, que l'on ne juge pas assez grave ou dangereuse pour la réprimer par des moyens plus violents ${ }^{30}$ ». Le manque de sérieux avec lequel les critiques abordent les composantes marginales de Refus global est en ce sens révélateur du statut qu'ils leur octroient, soit celui $d^{\prime}$ «œuvres encore inégales ${ }^{31} »$ ou de «recherche[s] assez égarée[s] qui n'en demeure[nt] pas moins sympathique[s] ${ }^{32} »$, œuvres de jeunesse donc, curieuses, expérimentales ou divertissantes, mais sans réel intérêt pour l'histoire. Ce statut d'œuvres «mineures» est par ailleurs renforcé par la proximité de ces composantes avec un texte «majeur» comme celui de Borduas qui ouvre le

25 Robert LaPalme, «Ces temps modernes», Le Canada, 21 décembre 1948, p. 4.

26 On ne peut certes pas affirmer que LaPalme rejetait l'art moderne; il a tout de même confié ne pas aimer «les mouvements». Voir Jean-François Nadeau, LaPalme. La caricature et autres sujets sérieux, Montréal, l'Hexagone, coll. «Entretiens», 1997, p. 103.

27 Nathalie Heinich, Le triple jeu de l'art contemporain, p. 218.

28 Ibid., p. 218-219.

29 Roger Duhamel, «Notes de lecture. "Refus global"», p. 4.

30 Chaïm Perelman et Lucie Olbrechts-Tyteca, Traité de l'argumentation. La nouvelle rhétorique, Bruxelles, Éditions de l'Université de Bruxelles, coll. «Euvres de Chaïm Perelman», 1988 [1958], p. 276.

31 Lafcadio, «L'underground de l'esthétique. "Refus global"», p. 4.

32 Roger Duhamel, «Notes de lecture. "Refus global”», p. 4. 
recueil et qui sera, lui, pris au sérieux, en fonction de critères non plus esthétiques, mais éthiques.

\section{LE CONGÉDIEMENT DE BORDUAS: NEEUD DE LA RÉCEPTION}

Au contraire de ceux que la critique nomme les «jeunes» membres du groupe, PaulÉmile Borduas jouit, en 1948, d'un statut de peintre et de professeur qui lui donne un ascendant sur ses élèves et sur ses admirateurs. D’où le fait que Gérard Pelletier, et la critique en général, condamne plus sévèrement encore les idées avancées dans le texte éponyme, idées qu'il qualifie, cette fois, du «comble de la loufoquerie et $d^{\prime}$ une loufoquerie mille fois plus dangereuse que l'autre ${ }^{33} »$. C'est d'ailleurs, faut-il le rappeler, en raison "[d]es écrits et [d]es manifestes qu'il publie, ainsi que [de] son état d'esprit [qui] ne sont pas de nature à favoriser l'enseignement que [le gouvernement] v[eut] donner ${ }^{34}$ » que Borduas est renvoyé de son poste de professeur à l'École du meuble un mois à peine après la parution de Refus global. Dans la lettre qui officialise le congédiement, le sous-ministre évoque plus spécifiquement une «conduite et [des] écrits incompatibles avec la fonction d'un professeur dans une institution d'enseignement de la province de Québec ${ }^{35}$ ». Ainsi, comme le fait remarquer Harry Bernard du Courrier de Saint-Hyacinthe, ce n'est pas simplement le caractère antinational ou anticlérical des propos contenus dans «Refus global» qui est à l'origine du renvoi, mais l'inconvenance de ceux-ci dans le cadre de la fonction assumée par Borduas: "Qu'un gogo quelconque s'amuse à pareil anticléricalisme de commis-voyageur [...], cela le regarde. S'il s'agit, comme dans le cas de Borduas, d'un homme appelé à former la jeunesse, à marquer un enseignement, il y a une différence ${ }^{36}$.»

La focalisation du discours critique sur le texte éponyme au détriment du reste du recueil s'explique donc avant même le congédiement, par le poste d'influence occupé par Borduas (ce dont ne jouissaient pas encore les autres signataires), lequel fait en sorte que lui seul portera la responsabilité et subira les conséquences de la prise de position collective. Par ailleurs, après le renvoi, le recueil se verra éclipsé par les débats que suscite la décision gouvernementale, lesquels remplaceront, dans la couverture médiatique, les commentaires sur l'œuvre; le texte éponyme lui-même n'étant plus guère désigné que comme l'œuvre «à l'origine de l'affaire ${ }^{37}$ ». Le renvoi de Borduas provoque en effet un soulèvement dans le milieu intellectuel: des lettres ouvertes condamnent cette décision «au nom de la démocratie [et des] libertés

33 Gêrard Pelletier, «Deux âges, deux manières», p. 8.

34 Extrait de la lettre du sous-ministre Gustave Poisson au directeur de l'École du meuble, Jean-Marie Gauvreau, annonçant la suspension de Borduas. Celle-ci a été reproduite dans plusieurs articles, dont «Borduas renvoyé. De l'École du meuble» (Le Devoir, 18 septembre 1948, p. 3) et «M. Borduas est mis à la porte» (Le Canada, 18 septembre 1948, p. 2).

35 Lettre contenue dans le Fonds d'archives Jean-Charles-Doyon, Bibliothèque et Archives nationales du Québec, boîte 2006-10-001/294. Je souligne.

36 Harry Bernard, «Le cas Borduas», Le Courrier de Saint-Hyacinthe, 24 septembre 1948, p. 3.

37 André Laurendeau, «Blocs-notes. Intervention politique», Le Devoir, 23 septembre 1948, p. 1. 
individuelles ${ }^{38}$ » et fustigent « un gouvernement du cadenas ${ }^{39}$ » qui «soume[t] au dirigisme intellectuel nos esprits créateurs ${ }^{40}$ ». André Laurendeau, qui n'a pas commenté la parution du recueil et qui se dissocie des propos de Borduas, y voit néanmoins une occasion de dénoncer $\mathrm{l}^{\prime}$ «intrusion cavalière de la politique [...] dans nos écoles ${ }^{41} »$. En somme, cette décision témoigne à la fois de la frontière encore poreuse entre champ culturel et champ du pouvoir à la fin des années 1940, laquelle rend possible une telle décision et, par les réactions qu'elle provoque, de l'autonomisation en cours, alors que certains réclament, en faveur de Borduas, l'intervention des institutions littéraires en place (l'Académie canadienne-française présidée par Victor Barbeau ${ }^{42}$ et la Société des poètes canadiens-français ${ }^{43}$ ).

L'événement que constitue le renvoi de Borduas à l'intérieur de la réception de Refus global a donc un double effet. D'une part, il éclipse l'œuvre: près de la moitié des articles recensés portent davantage sur ce qui est devenu le "cas ${ }^{44}$ » ou «l'affaire ${ }^{45}$ » Borduas que sur l'œuvre elle-même, que les critiques confinent au statut de cause du congédiement. D'autre part, le renvoi condense le discours autour de la figure de Borduas : ses défenseurs en viendront d'ailleurs à mettre en place, déjà en 1948, les éléments du mythe Borduas, lequel, «[p]rivé de ses sources de subsistance normales, abominablement maltraité jusqu'ici par l'ingratitude d'une patrie lymphatique [et vivant dans des] conditions de vie inhumaines ${ }^{46} "$, se verra forcé à l'exil. Le renvoi de Borduas constitue ainsi une nouvelle preuve, s'il en faut, de l'efficacité de la censure et des sanctions pour la consécration des œuvres, consécration qui a ici pour corollaire la relégation du recueil dans l'ombre de son texte éponyme.

\section{QUAND LES AUTEURS S'EN MÊLENT...}

La rhétorique laudative de la dernière citation concernant Borduas suggère à FrançoisMarc Gagnon qu'«il s'agit probablement de la prose colorée de Claude Gauvreau ${ }^{47}$ ». Gauvreau et d'autres membres du groupe participent en effet activement à la réception de leur œuvre et à la défense de Borduas en envoyant aux journaux des lettres ouvertes à teneur polémique. Prenant à partie la critique qu'ils accusent de faiblesse, d'opportunisme et d'incompétence, ils font preuve d'une arrogance et d'une intransigeance qui ont plutôt pour effet de les discréditer eux-mêmes. La polémique qui oppose les signataires de deux lettres collectives (Jean-Paul Riopelle, Maurice

38 Bernard Morisset, «Nous sommes avec vous, Borduas!», Le Canada, 2 octobre 1948, p. 4.

39 [S. a.], "Autour et alentour. La poésie devra peut-être se défendre, elle aussi », Le Canada, 22 septembre 1948 , p. 4.

40 [S. a.], "Garder notre élite chez nous», Le Canada, 23 septembre 1948, p. 4.

41 André Laurendeau, «Blocs-notes», p. 1.

42 P. E. Vadnais, «On devrait protester», Le Canada, 24 septembre 1948, p. 4.

43 [S. a.], "Autour et alentour. La poésie devra peut-être se défendre, elle aussi», p. 4.

44 [S. a.], "Après le "Refus"... Une enquête sur le cas de Borduas», Le Canada, 24 septembre 1948, p. 2.

45 [S. a.], «Les automatistes s'élèvent contre l'affaire Borduas», Le Petit Journal, 19 septembre 1948, p. 26.

46 Un automatiste, «Borduas devra-t-il s'exiler?», Le Canada, 20 mai 1949, p. 4.

47 François-Marc Gagnon, Paul-Émile Borduas (1905-1960). Biographie critique et analyse de l'œuvre, Montréal, Fides, 1978, p. 275. 
Perron, Magdeleine Arbour, Pierre Gauvreau et Françoise Riopelle) au chroniqueur Gérard Pelletier et à Jacques Dubuc est la plus représentative de cet «impossible dialogue ${ }^{48}$ » qui règne entre les automatistes et la critique et qui mettra fin à la couverture médiatique entourant Refus global un an à peine après sa parution.

Alors que Pelletier et Dubuc ont chacun fait paraître, dans Le Devoir, un texte proposant deux évaluations divergentes de la valeur des diverses composantes du recueil ${ }^{49}$ et qu'une discussion sur le sujet est sur le point de s'amorcer, les automatistes interviennent: «Nous croyons nécessaire, avant que ne s'engage définitivement une polémique [...], de dénoncer la critique qui a failli, on ne peut mieux, devant REFUS GLOBAL, à adopter une position permettant de discuter l'essentiel de la question ${ }^{50}$.» S'ensuit une liste d'arguments que les automatistes «refus[ent] d'entendre», minant ainsi la discussion et révélant leur manque de confiance envers les critiques, auxquels ils reprochent autant de ne pas adhérer aux propositions de Refus global que de ne pas les condamner totalement. Si, dans sa première réponse, Pelletier «repren[d] point par point ${ }^{51}$ » les questions et reproches soulevés par ses interlocuteurs pour clarifier son point de vue de manière plutôt posée, la seconde missive du collectif automatiste reçoit une réplique plus cinglante. Pelletier n'hésite alors pas à confronter les automatistes à leur propre contradiction et à l'impasse dans laquelle ils conduisent la discussion: «Voilà qui est clair. On vous invite à parler... mais on ne vous lira que pour la forme puisqu'on est convaincu d'avance (quelle confiance, quelle brûlante fraternité humaine!) que vous direz des sottises ${ }^{52}$.» Cet article sous-titré, de façon éloquente, «Dernier appel à la patience de nos lecteurs» témoigne de l'animosité qui existe entre les automatistes et la critique, laquelle compte pour beaucoup dans le silence qui finit par s'installer sur l'œuvre. La critique, parfois apeurée ${ }^{53}$, parfois rebutée par cette attitude intempestive, ne semble en effet plus vouloir traiter avec les automatistes, comme l'illustre une autre caricature de Robert LaPalme, parue en avril 1949, dans laquelle deux critiques murmurent à l'arrivée des automatistes au Salon du printemps: «Chut! chut! Voilà les automatistes ${ }^{54}$ !»

Moins lié au contexte qu'à l'attitude de certains individus, ce dernier facteur ayant contribué à l'effacement du recueil dans l'histoire culturelle met tout de même en lumière les effets d'une presse où les polémiques étaient encore chose courante, mais où les journalistes avaient toujours le dernier mot $-c^{\prime}$ est Pelletier qui met fin à cet «impossible dialogue» et interrompt la réception de Refus global.

48 Gérard Pelletier, «L'impossible dialogue. Dernier appel à la patience de nos lecteurs», Le Devoir, 20 novembre 1948, p. 10.

49 Pelletier se montre ouvert aux expérimentations des jeunes, mais condamne, on l'a vu, les idées de Borduas («Deux âges, deux manières», p. 8), alors que Dubuc défend au contraire Borduas et trouve «détestabl[e]» l'attitude des jeunes («Courrier sans confidences», Le Devoir, 30 octobre 1948, p. 5).

50 Jean-Paul Riopelle, Maurice Perron, Magdeleine Arbour, Pierre Gauvreau et Françoise Riopelle, «Les surréalistes nous écrivent», Le Devoir, 13 novembre 1948, p. 9.

51 Gérard Pelletier, «Notre réponse aux surréalistes», Le Devoir, 13 novembre 1948, p. 9.

52 Gérard Pelletier, «L'impossible dialogue», p. 10.

53 Agnès Lefort, par exemple, après une déclaration allant à l'encontre des idées du groupe, s'empresse d'ajouter n'être "pas intéressée à une polémique» avec les automatistes. Voir Marcel Gagnon, «La femme peintre Agnès Lefort est bien loin de croire à l'automatisme», Le Canada, 28 octobre 1948, p. 3.

Robert LaPalme, «Salon du printemps», Le Canada, 19 avril 1949, p. 4. 
En somme, l'étude de la première réception de Refus global a permis de mettre au jour les facteurs qui ont contribué, dès sa parution, à former le discours dominant sur l'œuvre, c'est-à-dire à resserrer le discours autour du texte éponyme (notamment en ce qui concerne le statut de son auteur) et à lui conférer un caractère subversif (en fonction non pas de son contenu, mais de sa conséquence: le congédiement de son auteur). Elle a aussi révélé ce qui a participé à l'effacement du recueil dans l'histoire, soit son caractère expérimental sur les plans matériel, disciplinaire et formel, ainsi que l'attitude de ses "piètres défenseurs", comme le soutient Jacques Dubuc ${ }^{55}$. Le cas de Refus global permet ainsi d'éclairer les tensions qui existaient, dans le champ culturel des années 1940, entre les diverses disciplines et entre des modes de lecture qui opposaient généralistes et spécialistes; lecture éthique et esthétique; critères de l'art moderne et de l'art traditionnel.

Plus largement, l'étude de la première réception de Refus global permet de réévaluer le topos de la rupture qui, à compter des années 1960, lui a été accolé comme œuvre marquant l'entrée du Québec dans la modernité culturelle. D’emblée, sur le plan esthétique, ce n'est pas le texte éponyme qui bouleverse le plus radicalement les attentes du public des années 1940, mais la matérialité de l'œuvre, sa pluridisciplinarité et les propositions esthétiques, tels les textes de Claude Gauvreau, qui correspondent difficilement aux schèmes interprétatifs et aux compétences des critiques de l'époque. Or ces composantes, tournées en dérision, minorées et soumises à un report de jugement, ne seront pas retenues dans l'histoire. Par ailleurs, le texte éponyme, aux dires de certains critiques, «n'est rien de bien neuf ${ }^{56}$ »; les idées antinationales et anticléricales qu'il contient ont déjà été énoncées par d'autres et sa forme, celle du pamphlet, ne bouleverse pas les conventions ${ }^{57}$. Aussi ce qui fait réellement «événement» et qui crée la rupture, c'est bien davantage le renvoi de Borduas que la parution du texte qui, somme toute, a donné lieu à peu de commentaires approfondis. La décision gouvernementale ainsi que les débats qui s'ensuivirent ont en effet fortement contribué à donner à «Refus global» le caractère subversif grâce auquel il serait relu postérieurement (notamment au moment de la mort de Borduas en 1960) en fonction du "grand récit» qui oppose la Grande Noirceur à la Révolution tranquille ${ }^{58}$. Sa dialectique qui condamne le passé au nom du présent et du futur

55 Jacques Dubuc, "La peau du lion et l'âne. En marge du "Refus global" et de ses piètres défenseurs», Le Devoir, 4 décembre 1948, p. 13. Dubuc fait également remarquer que les membres du groupe ayant signé les lettres de protestation envers la critique «sont loin d'être les plus significatifs parmi ceux qui ont endossé Refus global».

56 Lafcadio, «L'underground de l'esthétique», p. 4.

57 Michel Biron affirme d'ailleurs que le ton lui-même ne serait pas nouveau: il s'agit de celui du sermon. L'efficacité performative du texte de Borduas reposerait en cela sur l'usage de cette forme traditionnelle au profit d'un propos résolument moderne et iconoclaste. Michel Biron, «Distance du poème. Gilles Hénault et Refus global", Études françaises, vol. XXXIV, nos 2-3, automne-hiver 1998, p. 113-124.

58 Sur cette question du "grand récit» ou du «récit commun» entourant la Révolution tranquille et l'entrée dans la modernité du Québec, voir notamment Jocelyn Létourneau, "Le "Québec moderne". Un chapitre du grand récit collectif des Québécois», Discours social/Social Discourse, vol. IV, nos $1-2$, hiver-printemps 1992, p. 63-88; Micheline Cambron, «Raconter l'histoire de la littérature québécoise», Littérature, nº 124, 
(«Fini l'assassinat massif du présent et du futur à coup redoublé [sic] du passé ${ }^{59}$ ») et qui annonce l'avènement d'une nouvelle ère («nous entrevoyons l'homme libéré de ses chaînes inutiles ${ }^{60}$ ») épouse en effet parfaitement ce récit quelque peu manichéen et triomphaliste qui se mettra en place dans les années 1960. Cette réappropriation du texte par l'historiographie du Québec moderne aura pour conséquence de compléter le processus métonymique caractérisant la réception du manifeste automatiste: le tout ayant cédé le pas à la partie, Refus global est devenu «Refus global».

décembre 2001, p. 81-97; et Martine-Emmanuelle Lapointe, Emblèmes d'une littérature. Le libraire, Prochain épisode et L'avalée des avalés, Montréal, Fides, coll. «Nouvelles études québécoises», 2008, 357 p.

59 Paul-Émile Borduas et autres signataires, «Refus global», Refus global, Saint-Hilaire, Mithra-Mythe, 1948, p. 11.

60 Paul-Émile Borduas et autres signataires, «Refus global», p. 15. 